\title{
Proportional Analysis of Pig Kidney Arterial Segments: Differences from the Human Kidney
}

\author{
MARCO PEREIRA-SAMPAIO, D.V.M., Ph.D., ${ }^{1}$ LUCIANO ALVES FAVORITO, M.D., Ph.D., ${ }^{2}$ \\ ROBERT HENRY, D.V.M., Ph.D., ${ }^{3}$ and FRANCISCO J.B. SAMPAIO, M.D., Ph.D. ${ }^{2}$
}

\begin{abstract}
Purpose: To present a systematic study and a proportional analysis of the arterial segments of the pig kidney. Materials and Methods: Sixty-one three-dimensional endocasts of the arterial segments of pig kidneys were studied. Each segment was injected with a resin of a different color. Cavalieri's principle was used to calculate the volume of each renal segment, and these results were compared with the results from the point-counting planimetry method used on photographs of pig-kidney surfaces.

Results: Two to five renal segments were observed. Division into two segments, a cranial and a caudal, was the most common (42.62\%). The renal volume ranged from 101 to $173 \mathrm{~cm}^{3}$ (mean $130.85 \mathrm{~cm}^{3}$ ). The cranial segment was present in 39 of the 57 casts $(68.42 \%)$. It presented the greatest median value of proportional area $\mathbf{5 0 . 0 0 \%}$ ) and also the greatest maximum value of proportional area, accounting for as much as $\mathbf{7 4 . 0 4 \%}$ of the total kidney area. The ventral segment, which was found in 20 of the 57 casts $(35.09 \%)$, presented the lowest median value of proportional area $(13.87 \%$ ) and showed the most variation in area (coefficient of variation $\mathbf{7 2 . 8 9 \%}$ ). There was no significant statistical difference between the segmental areas as evaluated by Cavalieri's principle and by the point-counting planimetry method.

Conclusions: The distribution and size of the renal-arterial segments in pigs are not similar to those of the human kidneys. Therefore, this information must be taken into account by practitioners of urologic training or ablation using pigs as the animal model, as the structure of the porcine arterial segments cannot be transposed to humans.
\end{abstract}

\section{INTRODUCTION}

$\mathbf{T}$ IHE DISTRIBUTION OF THE INTRARENAL ARTERIES was described a long time ago in man and divided the renal parenchyma into anatomic segments. ${ }^{1}$ Several articles followed describing the arterial segmentation ${ }^{2-5}$ and the proportional surface areas of the segments. ${ }^{6,7}$

The pig kidney has been used as a model for many urologic procedures because of its resemblance to the human kidney. Although some studies on collecting-system and intrarenal-artery distribution have been described, none presents an analysis of the proportional areas of the segments. ${ }^{8-10}$ The pig has been the favorite animal model for training and experimental research in urology, including many studies on laparoscopic total and partial nephrectomy, hemostasis techniques, and, more recently, ablative technologies, including radiofrequency ablation (RFA) and cryoablation. ${ }^{11-14}$ Radiofrequency energy causes coagulative necrosis, which is later replaced by inflammation and fibrosis. In animal studies, parenchymal thrombosis and coagulation are seen after RFA ablation. The main limitation of RFA is the challenge of performing centrally located tumor excisions, which carries a high risk of collecting-system injury. ${ }^{15}$

Treatment for small renal tumors has been evolving from radical nephrectomy to nephron-sparing techniques, including ablation. ${ }^{16,17}$ Although cryotherapy and RFA are increasingly being applied clinically, there is a need for further research in this field. Other noninvasive tumor ablation techniques, such as high-intensity focused ultrasound, are still at an experimental stage. ${ }^{17}$ Laparoscopic partial nephrectomy and ablative techniques to treat renal masses may present complications associ-

\footnotetext{
${ }^{1}$ Department of Morphology, Fluminense Federal University, Niteroi, Brazil.

${ }^{2}$ Department of Anatomy and ${ }^{4}$ Urogenital Research Unit, State University of Rio de Janeiro, Rio de Janeiro, Brazil.

${ }^{3}$ Department of Comparative Medicine, University of Tennessee, Knoxville, Tennessee.
} 
ated with injury to important renal structures, which are not yet well understood. ${ }^{13}$ Some authors have been investigating the effects of ablation with cryosurgery or RFA on adjacent vital renal structures in a porcine model. ${ }^{12-14}$ Therefore, a comprehensive knowledge of the proportional areas of the arterial segments would be important for evaluating the extension of experimental lesions in pigs.

The aim of this study was to analyze pig kidney segmentation and the proportional area of each segment, as measured on polyester resin endocasts of the arterial vasculature.

\section{MATERIALS AND METHODS}

Our material consisted of unfixed kidneys taken from adult mixed-breed Duroc and Large-White farm pigs slaughtered at 140 days of age and weighing from 60 to $80 \mathrm{~kg}$ (mean $72 \mathrm{~kg}$ ) obtained from a slaughterhouse. The institutional animal review committee approved the research protocol. Sixty-one three-dimensional polyester resin endocasts of the intrarenal arterial tree (30 pairs and 1 right kidney) were prepared for study from 31 adult pigs of both sexes.

A segmental artery is defined as a primary or secondary branch of the main renal artery that can be identified and isolated outside the hilum. ${ }^{7,17}$ Colored polyester resin was injected into each segmental branch, with each segment receiving a different color of resin. The injections were performed according to a previously described technique. ${ }^{5,7,10,18,19}$ After the injections, the kidneys were preserved by submersion in a $10 \%$ formaldehyde solution for 48 hours, and both renal sides, dorsal and ventral, were photographed. A B-100 translucent Weibel $\operatorname{grid}^{20}$ was placed over the photographs in order to estimate the surface area of each segment using the point-counting planimetry method. ${ }^{7}$

As well, the Cavalieri's principle ${ }^{21}$ was used to obtain the real volume of the kidney and of each segment. The kidneys were sectioned transversally in 1-cm slices. The B-100 translucent Weibel grid was placed over the caudal surfaces of all slices and used for the point-counting planimetry method (Fig.
1). Thus, $V$ (segment) $=t a(p) \Sigma P($ segment $) u^{3}$, where V (segment $)=$ segmental volume, $t=$ slice thickness, $a(p)=$ area associated with each point in the Weibel grid, $\Sigma$ P (segment) $=$ all points hitting each color in all slices, and $\mathrm{u}^{3}=$ the known three-dimensional unit used in the test system and in the thickness of slices $\left(\mathrm{cm}^{3}\right)$.

Statistical analysis of the arterial segments was made considering the mean, the standard deviation, and the coefficient of variation. Fisher's test was used to compare the results obtained by Cavalieri's principle with those of the point-counting planimetry method used on photographs of kidney surfaces.

\section{RESULTS}

The total renal volume determined using Cavalieri's principle ranged from 101 to $173 \mathrm{~cm}^{3}$ (mean $130.85 \mathrm{~cm}^{3}$ ) and that obtained using the point-couting planimetry method ranged from 97 to $176 \mathrm{~cm}^{3}$ (mean $\left.134.48 \mathrm{~cm}^{3}\right)(\mathrm{P}=\mathrm{NS})$. The primary division of the renal artery into a cranial and a caudal branch was found in all kidneys with segmental division. In 4 of 61 casts $(6.55 \%)$, the renal artery did not branch outside the renal hilum, and, therefore, these kidneys did not present any segmentation.

In the kidneys that presented primary or secondary branches of the renal artery outside the renal hilum, two to five renal segments were identified. The arrangement of the segments was amply varied and was not symmetrical. Segmental bilateral symmetry was found in only $26.67 \%$ of the cases ( 8 pairs of casts). The division into two segments, cranial and caudal (Fig. 2) was the most common arrangement $(42.62 \%)$. The different types of segmental arrangements and their frequencies are listed in Table 1.

Cranial segment. The cranial division of the renal artery usually divided inside the parenchyma. In these cases, which accounted for 39 of 57 casts $(68.42 \%)$, only one segment in the cranial pole was found, and its area ranged from $29.06 \%$ to $74.04 \%$ of the total kidney area (mean $50 \%$ ), with the vessel supplying both the dorsal and the ventral surfaces of the

A

B

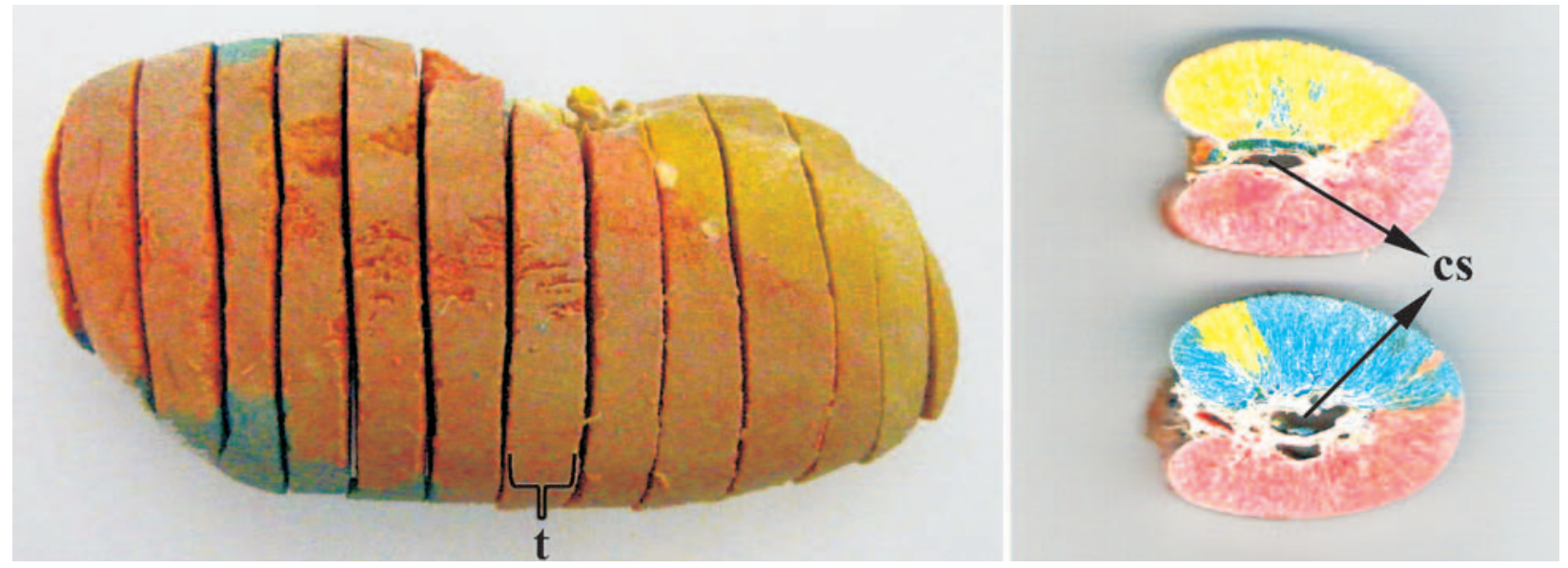

FIG. 1. Measurement according to Cavalieri's principle. (A) Ventral view of transversely sectioned pig kidney showing serial sections. $\mathrm{t}=$ slice thickness. (B) Caudal view of two slices, showing caliceal system (cs) transversally sectioned. 


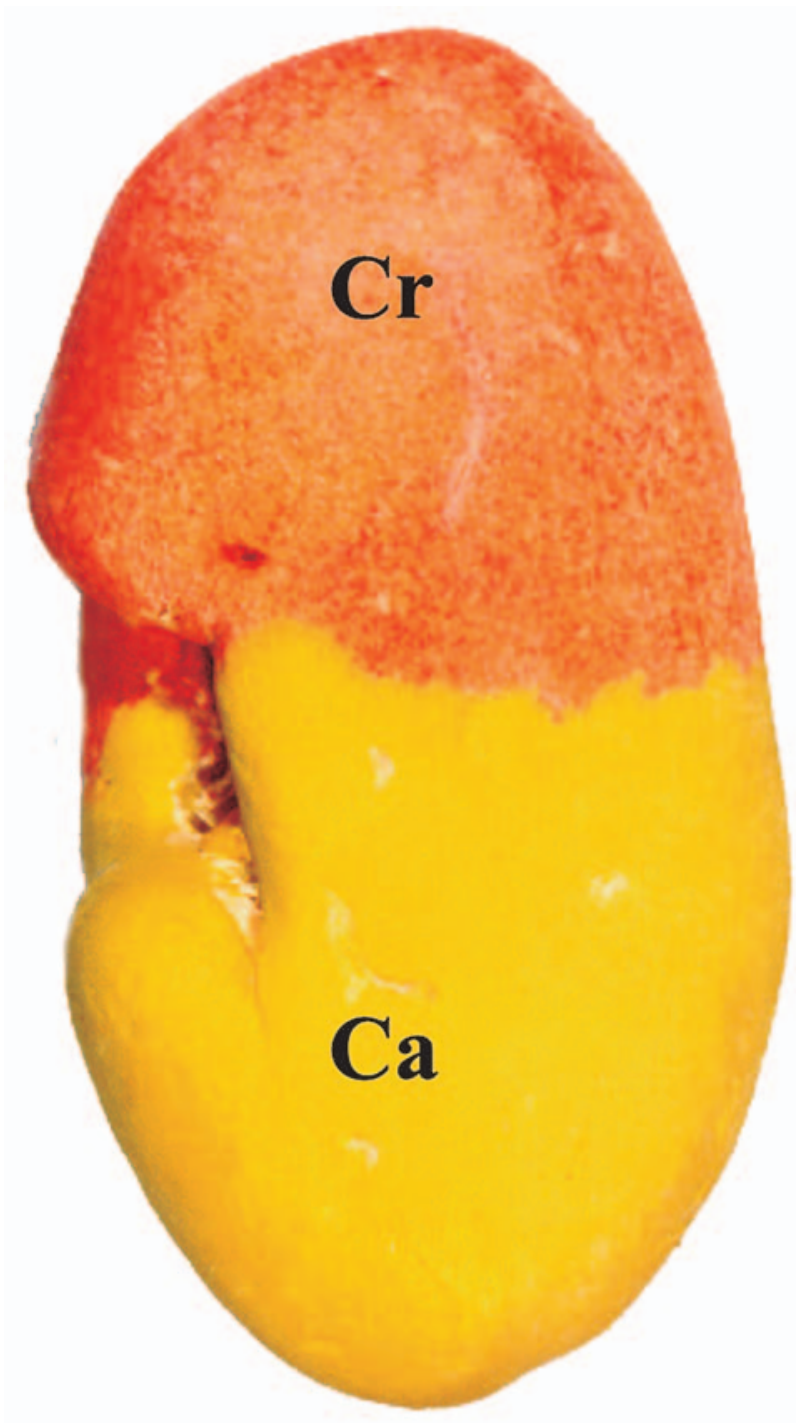

FIG. 2. Dorsal view of pig kidney with two arterial segments. $\mathrm{Cr}=$ cranial segment; $\mathrm{Ca}=$ caudal segment.

cranial pole. When the cranial division of the renal artery divided outside the renal hilum (31.58\%), the cranial pole presented a craniodorsal and a cranioventral segment, and their mean areas were $28.01 \%$ and $23.78 \%$ of the total kidney area, respectively.
Ventral segment. This segment was found in 20 of the 57 casts $(35.09 \%$ ), and its area ranged from $2.97 \%$ to $23.9 \%$ of the total kidney area (mean $13.87 \%$ ). The ventral segment received secondary branches from the cranial or caudal division of the renal artery or both.

Dorsal segment. In 9 of the 57 casts (15.79\%), a dorsal segment was identified, and its area ranged from $5.84 \%$ to $29.25 \%$ of the total kidney area (mean $16.67 \%$ ). The dorsal segment received secondary branches from the cranial or caudal division of the renal artery or both.

Caudal segment. The caudal division of the renal artery gave branches within the hilum in 55 of the 57 casts $(96.49 \%)$, so, in these cases, the caudal pole presented only a caudal segment, including dorsal and ventral surfaces. Its area ranged from $20.75 \%$ to $63.5 \%$ of the total kidney area (mean $41.57 \%$ ). However, in 2 of the 57 casts (3.51\%), the caudal pole received two segmental arteries: a ventral branch from the caudal division of the renal artery and a dorsal branch from the cranial division of the renal artery. Therefore, in these casts, a caudodorsal and a caudoventral segment were found. Their mean areas were $24.03 \%$ and $27.79 \%$ of the total kidney area, respectively.

Table 2 lists the statistical analysis of the proportional surface areas of the segments.

\section{DISCUSSION}

Although the point-counting planimetry method is simple and reproducible and may be used in medical practice, it is not ideal for measuring the area of three-dimensional specimens. Therefore, a method for measurement characterized by a strong property of unbiasedness was desired. Cavalieri's principle does not require any unrealistic assumptions about the segment's shape and orientation; hence, it was used..$^{21}$ By comparing the results obtained by these two methods (Fisher's test), we verified their similar accuracy. Hence, the point-counting planimetry method, which is a simple and reasonable technique for quantifying renal segments, can be used in clinical practice.

A cranial segment was present in 39 of the 57 casts $(68.42 \%)$ and always included the dorsal and ventral surfaces of the kidney. This segment presented the greatest median value of proportional area $(50 \%)$ and the greatest maximum value of proportional area, accounting for as much as $74.04 \%$ of the total kidney area. This is not the case in humans, where the corresponding superior (apical) segment is not so important, with its area ranging from $5.17 \%$ to $27.02 \%$ of the total kidney area

Table 1. Arrangement of Pig Kidney Arterial Segments

\begin{tabular}{llr}
\hline $\begin{array}{l}\text { Number of } \\
\text { segments }\end{array}$ & \multicolumn{1}{c}{ Arrangement of segments } & $\begin{array}{c}\text { Percent of } \\
\text { total }\end{array}$ \\
\hline 2 & Cranial and caudal & 42.62 \\
3 & Cranial, ventral, and caudal & 13.11 \\
& Cranioventral, cranidorsal, and caudal & 11.48 \\
4 & Cranioventral, cranidorsal, ventral, and caudal & 8.20 \\
& Cranial, dorsal, ventral, and caudal & 8.20 \\
& Cranioventral, cranidorsal, caudoventral, and caudodorsal & 3.28 \\
& Cranioventral, cranidorsal, dorsal, and caudal & 3.28 \\
5 & Cranioventral, cranidorsal, dorsal, ventral, and caudal & 3.28 \\
\hline
\end{tabular}


Table 2. Results of Statistical Evaluation of Arterial Segment Proportional Analysis by Cavalieri’s Principle

\begin{tabular}{lccccccccc}
\hline & & & & & & & & $\begin{array}{c}\text { Renal volume } \\
\left(\mathrm{cm}^{3}\right)\end{array}$ \\
\hline No. & 39 & 18 & 18 & 20 & 9 & 55 & 2 & 2 & 61 \\
Mean & 50 & 28.01 & 23.78 & 13.87 & 16.67 & 41.57 & 24.03 & 27.79 & 130.85 \\
SD & 11.31 & 9.15 & 9.24 & 10.11 & 9.03 & 10.1 & 4.14 & 8.2 & 17.46 \\
CV & 22.62 & 32.67 & 38.86 & 72.89 & 54.17 & 24.3 & 17.23 & 29.51 & 13.34 \\
Minimum & 29.06 & 13.67 & 5.96 & 2.97 & 5.84 & 20.75 & 21.1 & 21.99 & 101 \\
Maximum & 74.04 & 42.35 & 41.61 & 23.9 & 29.25 & 63.5 & 26.95 & 33.59 & 173 \\
\hline
\end{tabular}

Abbreviations: $\mathrm{SD}=$ standard deviation; $\mathrm{CV}=$ coefficient of variation.

(mean $13.02 \%){ }^{7}$ In 18 cases $(31.58 \%)$, the cranial division of the renal artery divided outside the hilum and gave rise to a craniodorsal and a cranioventral segment. In these cases, the craniodorsal segment presented a greater median value of proportional area than the cranioventral segment.

The ventral segment, which was found in 20 of the 57 casts $(35.09 \%)$, presented the lowest mean value of proportional area $(13.87 \%)$ and also the most varied segment regarding its area (coefficient of variation $72.89 \%$ ). In humans, the ventral (anterior) segment is divided into an anterosuperior segment (mean area $21.36 \%$ ) and an anteroinferior segment (mean area $17.18 \%$ ) in $61.2 \%$ of cases. On the other hand, in $38.8 \%$ of the cases, there was only one segmental artery to the ventral surface of the mid-kidney, this being the anterosuperior and the anteroinferior segment fused as the anterior (ventral) segment (mean area 28.44\%). In human kidneys, the superior (apical) segment presented the most varied segment regarding area, ${ }^{6}$ different from the pig kidney, where the apical artery was not found outside the hilum and did not give off an apical segment.

The dorsal segment was present in only $15.79 \%$ of cases. Both dorsal and ventral segments received secondary branches from the cranial or caudal division of the renal artery. The occurrence of these segments depended on the origin of their arteries. If these branches arise inside the hilum, kidneys will not have dorsal or ventral segments (Fig. 2) but a portion of the cranial or caudal segments. If these branches arise outside the parenchyma, the kidneys will have dorsal or ventral segments (Fig. 3). This information is important because it demonstrates that these segments are considerably different from those of humans, where the posterior (dorsal) segment occurs in $100 \%$ of the cases. ${ }^{7,18,19}$ Moreover, in the human kidney, the posterior segment has the greatest median value of proportional area $(33.76 \%)$ and the greatest maximum value of proportional area, accounting for as much as $52.93 \%$ of the kidney total area. ${ }^{7}$ This fact is important because demonstrates the significance of the posterior segmental artery in humans, which is very different from the situation in pigs.

The caudal segment was present in 55 of the 57 cases (96.49\%) and always included the dorsal and ventral surfaces of the caudal pole. This segment was supplied by the caudal division of the renal artery, which divided within the parenchyma to supply both the dorsal and the ventral surfaces of the kidney. In only 2 of the 57 casts $(3.51 \%)$ was a dorsal branch from the cranial division of the renal artery found outside the hilum that supplied the dorsal surface of the caudal pole. In these cases, the caudal pole presented a caudodorsal and a caudoventral segment. The corresponding segment in humans (infe- rior segment) occurs in $100 \%$ of the cases. Whereas in pigs, its area ranged from $20.75 \%$ to $63.5 \%$ of the total kidney area (mean $41.57 \%$ ), in humans, this segment is much smaller, ranging from $7.42 \%$ to $38.18 \%$ of the total kidney area (mean $22.65 \%)^{7}$

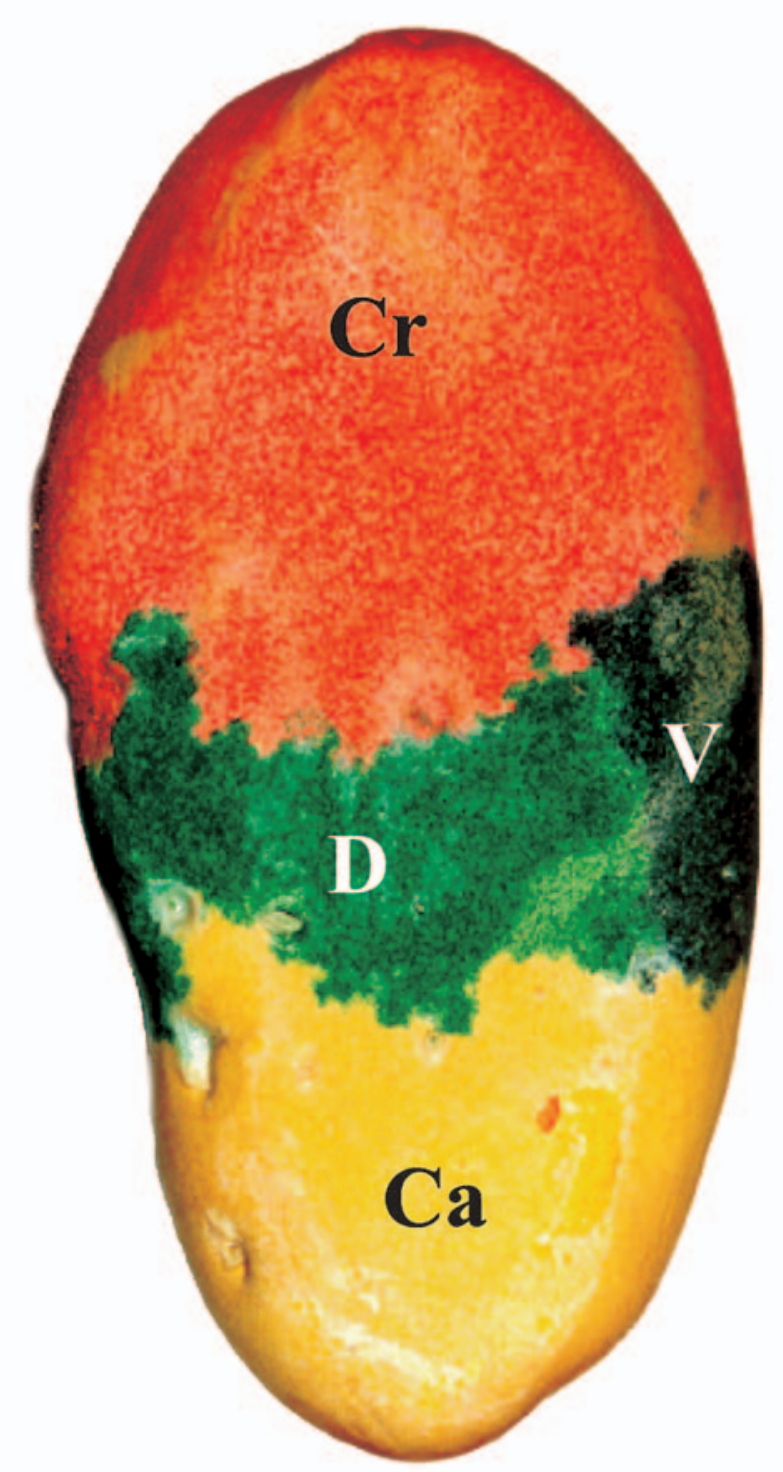

FIG. 3. Dorsal view of pig kidney with four segments: $\mathrm{Cr}=$ cranial, $\mathrm{V}=$ ventral, $\mathrm{D}=$ dorsal, $\mathrm{Ca}=$ caudal. 
The pig kidney presented two to five segments, and cranial and caudal segments were the most important. They were found in $100 \%$ of the cases and had the greatest median value of proportional area, with or without division. The human kidney presents four to five segments, ${ }^{1-3,5-7}$ of which the anterior and posterior segments are the most important. The pig renal artery divides into a cranial and a caudal branch in $93.4 \%$ of kidneys, ${ }^{10}$ whereas the primary division of human renal artery, when it is a single artery, is into an anterior and a posterior branch. ${ }^{5}$ This fact can explain the difference between the most important kidney arterial segments in pigs and humans, because the segmental division seems to depend on the primary division of the renal artery. Whereas in human kidney, the anterior (ventral) and posterior (dorsal) are the most important segments, ${ }^{7}$ in pig kidneys, these segments are not present in $64.91 \%$ and $84.21 \%$ of kidneys, respectively. The cranial and caudal segments are the most important segments in pig kidneys.

\section{CONCLUSION}

We believe that these data must be strongly considered by practitioners involved in experimental urologic procedures with pigs, because the arterial segmental pattern is not similar to that of the human kidneys. Therefore, the experimental findings concerning renal ablative techniques in the pig must not be translated directly to the clinical setting in humans.

\section{REFERENCES}

1. Graves FT. The anatomy of the intrarenal arteries and its application to segmental resection of the kidney. Br J Surg 1954;42:132.

2. Sykes D. The correlation between renal vascularisation and lobulation of the kidney. Br J Urol 1964;36:549.

3. Fine H, Keen EN. The arteries of the human kidney. J Anat 1966; 100:881.

4. Vondermark JS. Segmental anatomy of the kidney. Urology 1981;17:521.

5. Sampaio FJ, Arag o AH. Anatomical relationship between the intrarenal arteries and the kidney collecting system. J Urol 1990; 143:679.

6. Longia GS, Kumar V, Saxena SX. Surface projection of arterial segments in the human kidney. Acta Anat 1982;113:145.

7. Sampaio FJ, Schiavini JL, Favorito LA. Proportional analysis of the kidney arterial segments. Urol Res 1993;21:371.

8. Evan AP, Connors BA, Lingeman JE, Blomgren P, Willis LR. Branching patterns of the renal artery of the pig. Anat Rec 1996; 246:217.
9. Sampaio FJ, Pereira-Sampaio MA, Favorito LA. The pig kidney as an endourologic model: Anatomic contribution. J Endourol 1998;12:45.

10. Pereira-Sampaio MA, Favorito LA, Sampaio FJ. Pig kidney: Anatomical relationships between the intrarenal arteries and the kidney collecting system. Applied study for urological research and surgical training. J Urol 2004;172:2077.

11. Wilhelm DM, Ogan K, Saboorian MH, Napper C, Pearle MS, Cadeddu JA. Feasibility of laparoscopic partial nephrectomy using pledgeted compression sutures for hemostasis. J Endourol 2003; 17:223.

12. Slabaugh TK, Machaidze Z, Hennigar R, Ogan K. Monitoring radiofrequency renal lesions in real time using contrast-enhanced ultrasonography: A porcine model. J Endourol 2005;19:579.

13. Brashears JH 3rd, Raj GV, Crisci A, et al. Renal cryoablation and radio frequency ablation: An evaluation of worst case scenarios in a porcine model. J Urol 2005;173:2160.

14. Janzen NK, Perry KT, Han KR, et al. The effects of intentional cryoablation and radio frequency ablation of renal tissue involving the collecting system in a porcine model. J Urol 2005;173:1368.

15. Rubinstein M, Moinzadeh A, Colombo JR Jr, Favorito LA, Sampaio FJ, Gill IS. Energy sources during laparoscopic partial nephrectomy: Critical appraisal. Int Braz J Urol (in press).

16. Johnson DB, Solomon SB, Su LM, et al. Defining the complications of cryoablation and radio frequency ablation of small renal tumors: A multi-institutional review. J Urol 2004;172:874.

17. Aron M, Gill IS. Renal tumor ablation. Curr Opin Urol 2005; 15:298.

18. Sampaio FJ. Anatomical background for nephron-sparing surgery in renal cell carcinoma. J Urol 1992;147:999.

19. Sampaio FJ, Zanier JF, Aragao AH, Favorito LA. Intrarenal access: 3-Dimensional anatomical study. J Urol 1992;148:1769.

20. Weibel ER. Practical Methods for Biological Morphometry, vol 1. London: Academic Press, 1979.

21. Gundersen HJG, Bendtsen TF, Korbo L, et al. Some new, simple and efficient stereological methods and their use in pathological research and diagnosis. APMIS 1988;96:379.

Address reprint requests to:

Francisco J.B. Sampaio, M.D., Ph.D.

Urogenital Research Unit

State University of Rio de Janeiro

Av 28 de Setembro 87, fundos, predio da FCM, terreo Rio de Janeiro, Brazil 20551-030

E-mail: sampaio@urogenitalresearch.org brazjurol@uol.com.br

\section{ABBREVIATIONS USED}

$\mathrm{NS}=$ not significant; RFA $=$ radiofrequency ablation. 